\title{
DESIGN, MANUFACTURING AND ANALYSIS OF BIO-MEDICAL PARTS (3D PRINTED BONE AND TOOTH) USING ADDITIVE MANUFACTURING
}

\author{
Alapati Venkateswarlu ${ }^{1}$ and Adusumilli Adilakshmi ${ }^{2}$
}

\begin{abstract}
Present paper reports on the advances in further developing of Additive Manufacturing technology, as well as in related post processing, necessary to address the challenges presented by biomedical applications. Additive Manufacturing has solidly established itself not only in rapid prototyping but also in industrial manufacturing. Its success is mainly determined by a possibility of manufacturing components with extremely complex shapes with minimal material waste. Rapid development of Additive Manufacturing technologies includes processes using unique new materials, which in some cases is very hard or impossible to process any other way. Along with traditional industrial applications Additive Manufacturing methods are becoming quite successful in biomedical applications, in particular in implant and special tools manufacturing. Here the capacity of Additive Manufacturing technologies in producing components with complex geometric shapes is often brought to extreme. Certain issues today are preventing the Additive Manufacturing methods taking its deserved place in medical and biomedical applications. Present paper reports on the advances in further developing of Additive Manufacturing technology, as well as in related post processing, necessary to address the challenges presented by biomedical applications. Particular examples used are from Electron Beam Melting (EBM), one of the methods from the Additive Manufacturing family. Due to advancement of technology additive manufacturing has also extended its inclination in biomedical applications. We can replace broken bone with the 3D printed bone. Recently, scientists have successfully refilled the damage that occurred to human skull by using biodegradable material. Human tissues can also be developed by the growth of living cells over the requisite.

Key Words: Additive Manufacturing, Biomedical Applications, Biodegradable Material, Analysis, 3D Printed Bone
\end{abstract}

\section{INTRODUCTION}

1. ADDITIVE MANUFACTURING: Additive Manufacturing refers to a process by which digital 3D design data is used to build up a component in layers by depositing material. The term "3D printing" is increasingly used as a synonym for Additive Manufacturing. Additive Manufacturing is an appropriate name to describe the technologies that build 3D objects by adding layer-upon-layer of material, whether the material is plastic, metal, concrete or one day.....human tissue. Commonly for Additive Manufacturing technologies used are a computer, 3D modelling software (Computer Aided Design or $\mathrm{CAD}$ ), machine equipment and layering material. Once a CAD sketch is produced, the Additive Manufacturing equipment reads in data from the CAD file and lays downs or adds successive layers of liquid, powder, sheet material or other, in a layer-upon-layer fashion to fabricate a 3D object.

\footnotetext{
${ }^{1}$ Professor, Mechanical Engineering, V.R.Siddhartha Engineering College, Vijayawada, AP.

${ }^{2}$ Professor, Civil Engineering, P.V.P.Siddhartha Institute of Technology, Vijayawada, AP.
} 


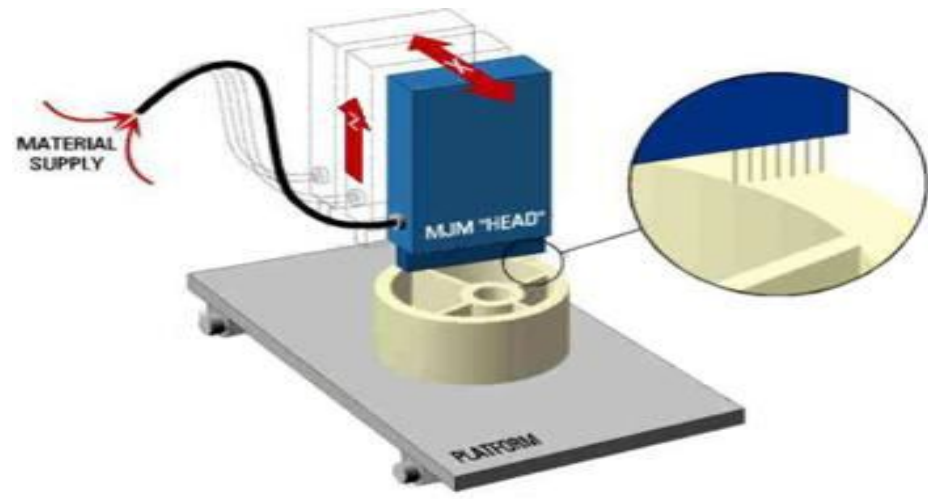

Fig1:The figure shows MJM(Multi-Jet Modelling) Printer

The term Additive Manufacturing encompasses many technologies including subsets like 3D Printing, Rapid Prototyping (RP), Direct Digital Manufacturing (DDM), layered manufacturing and additive fabrication.

Additive Manufacturing application is limitless. Early use of Additive Manufacturing in the form of Rapid Prototyping focused on preproduction visualization models. More recently, Additive Manufacturing is being used to fabricate end-use products in aircraft, dental restorations, medical implants, automobiles, and even fashion products. While the adding of layer-upon-layer approach is simple, there are many applications of Additive Manufacturing technology with degrees of sophistication to meet diverse needs including:

1. A visualization tool in design 2. A mean to create highly customized products for consumers and professionals alike 3 . A industrial tooling 4. To produce small lots of production parts 5. One day production of human organs.

At MIT, where the technology was invented, projects abound supporting a range of forward-thinking applications from multi-structure concrete to machines that can build machines; while work at Contour Crafting supports structures for people to live and work in.

Some envision Additive Manufacturing as a compliment to foundational subtractive manufacturing (removing material like drilling out material) and to lesser degree forming (like forging). Regardless, Additive Manufacturing may offer consumers and professionals alike, the accessibility to create, customize and/or repair product, and in the process, redefine current production technology. Whether simple or sophisticated, Additive Manufacturing is indeed amazing and best described in the adding of layer-upon-layer, whether in plastic, metal, concrete or one day...human tissue".

$1.1+\operatorname{MJM}$ ( Multi-Jet Modelling,Fig-1) is similar to an inkjet printer in that a head, capable of shuttling back and forth (3 dimensions- $\mathrm{x}, \mathrm{y}, \mathrm{z}$ )) incorporates hundreds of small jets to apply a layer of thermos polymer material, layer-by-layer.Multi Jet Modelling is one of the high-end solutions in the 3D printing market. This technology is unique because one large print head is used, which covers the full width of the building platform.

1.2 + FDM: Objects created with an FDM(Fig-2) printer start out as computer-aided design (CAD) files. Before an object can be printed, its CAD file must be converted to a format that a 3D printer can understand - usually .STL format. FDM printers use two kinds of materials, a modelling material, which constitutes the finished object, and a support material, which acts as a scaffolding to support the object as its being printed.

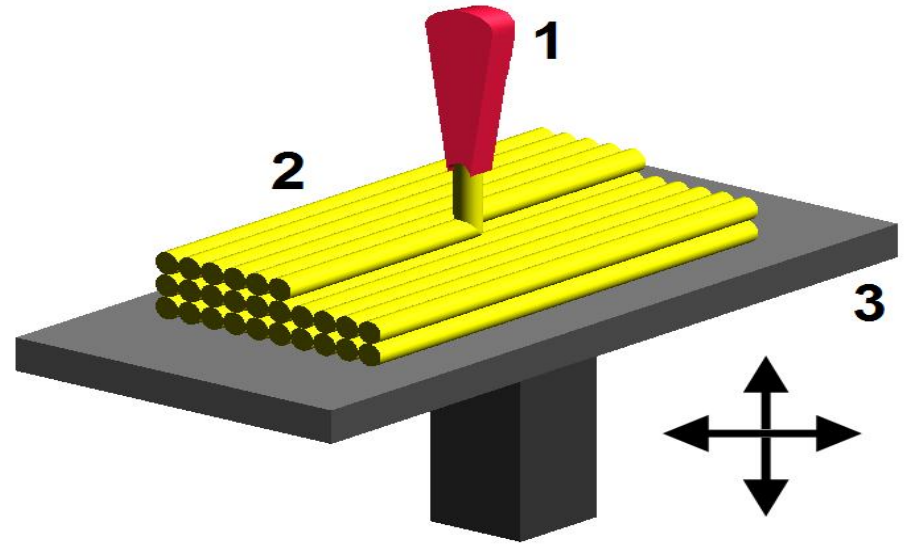

Fig 2:The figure shows FDP Printer

\section{FABRICATION OF EXTERNAL DEVICES}


There are two active biomedical application markets for integrated additive/subtractive manufacturing, primarily additive manufacturing, of external devices: those for hearing aid shells and for dental fixtures, including bridges. Envisiontec, working closely with the Freiburg Materials Research Center (FMF) that the WTEC panel visited in Germany, has actively pursued both the hearing aid and dental fixture markets using its Perfactory fabrication system. The Perfactory system uses photo polymerization to build structures. Instead of using a laser, however, the Perfactory system uses a light source that is masked using a grid of digital mirrors. The light source is underneath and shines up on the polymer. A system of digital mirrors in a $1280 \times 1024$ grid is used to mask the light shining on the polymer. If a mirror grid is open, the material underneath this mirror is completely polymerized and becomes part of the structure.

If a mirror grid is closed, no light shines on the material and this grid area becomes a void. If he mirror is partway open, the material is partially polymerized and becomes a support. The theoretical feature resolution of the Perfactory system is 32 microns, the length of one digital mirror. The Perfactory system builds at $15 \mathrm{~mm} /$ hour at a 50 -micron layer thickness and $25 \mathrm{~mm} /$ hour at a 100-micron layer thickness. The 32-micron feature size places the Perfactory system as the fabrication system with the highest feature resolution. The high resolution combined with rapid manufacturing speed make Envisiontec's Perfactory system well suited for making dental fixtures, including crowns, caps, and bridges. For these applications, the Perfactory system has been used to make moulds. Material is then cast into the mold to create the final application. The moulds are built at a layer thickness of 30 microns, with in-plane resolution of 32 microns. This again demonstrates the use of a finer resolution fabrication system for this biomedical application. The other European site visited by the WTEC panel that was pursuing dental manufacturing applications is TNO Industrial Technology in the Netherlands. Among the most successful biomedical dental applications are the Invisalign orthodontic custom braces by Align Technology, Inc., headquartered in Santa Clara, California. In this process, a dentist makes an impression, which is sent to Align Technologies. The impression is then scanned and the orthodontic appliances are made on a stereolithographic system. As a prime example of mass customization, over 100,000 patients have been treated with the Invisalign system. This clearly demonstrates the advantages of integrated additive/subtractive manufacturing to create custom, patient-specific anatomic products.

The other external biomedical device application for integrated additive/subtractive manufacturing is hearing aids. There is currently a $25 \%$ return rate due to improper fitting of hearing aid shells. To address these issues, Materialize in Belgium is collaborating with Phonak and Siemens to directly create STL files by scanning impressions of the inner ear. This project, entitled the Rapid Shell Modelling project, will not only save labor in creating a patient-specific hearing aid, but will also provide a digital archive of that patient's hearing aid shell in case the original shell is lost. Envisiontec is also targeting the hearing aid shell market with the Per factory system. In fact, Envisiontec estimates a market for 25 to 50 systems in Europe, a total of up to $€ 2$ million. Envisiontec has demonstrated the capability to build 20 hearing aid shells in 90 minutes. Both hearing aid shells and dental fixtures are maturing markets that demonstrate the clear advantage of integrated additive/subtractive manufacturing to create mass-customized anatomic devices.

\section{FABRICATION OF TISSUE ENGINEERING SCAFFOLDS}

Tissue engineering scaffolds represent the most risky, but at the same time most exciting, venture with the largest potential payoff for biomedical integrated additive/subtractive manufacturing applications over the next 10 to 30 years. Although a great deal of progress is currently being made fabricating tissue engineering scaffolds, the ultimate fabrication technology will reach goals 3-5 noted in the introduction to this chapter, that is, be able to fabricate hybrid biomaterial and cell/gene/protein structures over a range of size scales.Integrated additive/subtractive manufacturing will be the conduit through which biomaterial, biomechanical, and design engineering technologies are brought together with biological technologies of stem cell therapy, gene therapy, and protein therapy. As such, there will not be a separate scaffold seeded with cells or genes, but rather a hybrid scaffold/cell/gene structure fabricated by an integrated additive/subtractive manufacturing system.

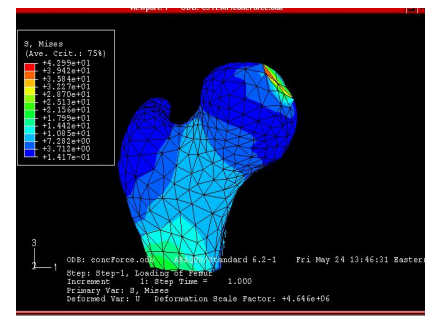

Fig 3:FEA analysis of Femur

In addition to direct biomaterial processing, many groups have also used SFF systems to create molds and then cast biomaterials directly into the moulds. Taboos et al. (2003) were able to use this technique to create scaffolds from both ceramic and polymer scaffolds, as well as to create discrete composite ceramic and polymer scaffolds. They were also able 
to create scaffolds with multiple pore scales and features down to five microns in size by combining SFF casting techniques with more traditional biopolymer processing techniques. Of the European sites the WTEC panel visited, three were fabricating calcium phosphate ceramic scaffolds using SFF. Isotis, a biomaterials company, has also created calcium phosphate ceramics using SFF molds created using the Envisiontec Per factory system. The University of Loughborough has created hydroxyapatite (HA) ceramic scaffolds using SLS.

\section{DSIGN AND FABRICATION}

The machine (Dimension 1200 es Machine) is used bone and Teeth Manufacturing, is provided with both the support material and model material cartridges placed inside it. The model material is cream in color and support material is brown in color this helps to easily identify the difference between support material and build material.

4.1 ACRYLONITRILE BUTADIENE STYRENE (ABS) PLUS: Acrylonitrile Butadiene Styrene (ABS) is an opaque thermoplastic and amorphous polymer. "Thermoplastic" (as opposed to "thermoset") has to do with the way the material responds to heat. Thermoplastics become liquid (i.e. have a "glass transition") at a certain temperature (221 degrees Fahrenheit in the case of ABS plastic). They can be heated to their melting point, cooled, and re-heated again without significant degradation. Instead of burning, thermoplastics like ABS liquefy which allows them to be easily injection molded and then subsequently recycled. ABS has a strong resistance to corrosive chemicals and/or physical impacts. It is very easy to machine and has a low melting temperature making it particularly simple to use in 3D printing on an FDM machine. ABS is also relatively inexpensive. All of these characteristics lead to ABS being used in a large number of applications across a wide range of industries. The most important mechanical properties of ABS are impact resistance and toughness. A variety of modifications can be made to improve impact resistance, toughness, and heat resistance. Specifications of ABS are given below.

\begin{tabular}{|l|l|}
\hline \multicolumn{1}{|c|}{ Property } & \multicolumn{1}{|c|}{ Value } \\
\hline Technical Name & Acrylonitrile butadiene styrene (ABS) \\
\hline Chemical Formula & $\left(\mathrm{C}_{8} \mathrm{H}_{8}\right)_{\mathrm{x}} \cdot\left(\mathrm{C}_{4} \mathrm{H}_{6}\right)_{\mathrm{y}^{\prime}} \cdot\left(\mathrm{C}_{3} \mathrm{H}_{3} \mathrm{~N}\right)_{\mathrm{z}}$ \\
\hline Tensile Strength (Type 1, 0.125",0.2"/min) & $36 \mathrm{MPa}$ \\
\hline Tensile Modulus (Type 1, 0.125",0.2"/min) & $2,300 \mathrm{MPa}$ \\
\hline Tensile Elongation (Type 1, 0.125",0.2"/min) & $4 \%$ \\
\hline Flexural Strength (Method 1,0.05"/min) & $52 \mathrm{MPa}$ \\
\hline Flexural Modulus (Method 1, 0.05"/min) & $2,200 \mathrm{MPa}$ \\
\hline Typical Injection Molding Temperature & $290-310^{\circ} \mathrm{C}$ \\
\hline Specific Gravity & 1.06 \\
\hline Dielectric Constant & $2.9-2.7$ \\
\hline
\end{tabular}

\subsection{Meshlab software}

We have taken X-ray of femur bone and tooth of human aged-32 years from a diagnostic centre and have been converted to .STL file using MESHLAB software and given as a input to 3D printer.

4.3 Machine Software:The software utilized by the DIMENSION1200 es machine is CATALYST 4.4 where the cad model after converting into .STL file format it is imported into machine software for the communication between the software and printer. The software is designed and provided with various options such layer resolution, model infill type (like solid, sparse- low density, sparse high density), STL scale, STL report of the cad model, orientation angle, STL simulation. After simulation of STL file it is added to PACK where it gives process parameters such as model material volume, support material volume, build time. The final step is proceed for printing by the machine.

\subsection{Orientation of bone}

We had made the bone in two orientations.1. 00 orientation 2. 900 orientation.

Generally on 3D printer we can make objects in any two above orientations. The difference in the two orientations is their strengths. In one orientation it may break through tension and in one orientation it may break through compression. The 
main moto in building this in two orientations is just to create awareness of using $3 \mathrm{~d}$ printing machine and we just created some models of human femur bone and teeth.

We have done the $3 \mathrm{~d}$ printing of femur bone by taking sample MRI scan image from diagnostic centre and have been converted into the .STL file format using MESH LAB software and by scaling the femur bone $3 \mathrm{~d}$ printing is completed.

We have scaled to 8 inches and made horizontally on the $3 \mathrm{~d}$ printer bed so that it can't break easily and has some fatigue failure which we found out using some mechanical tests.

We have used ABS (Acrylonitrile Butadiene Styrene). ABS is a thermoplastic resin commonly used for injection moulding applications. ABS Plastic is a copolymer of Acrylonitrile, Butadiene, and Styrene, and generally possess medium strength and performance at medium cost.

In general for $3 \mathrm{~d}$ printing of bones generally Titanium is used. Which has highest strength, durability. But Titanium is of higher cost. Just we made a prototype of it. In general bone inner structure has lipids which can be grown by living cells under proper conditions.
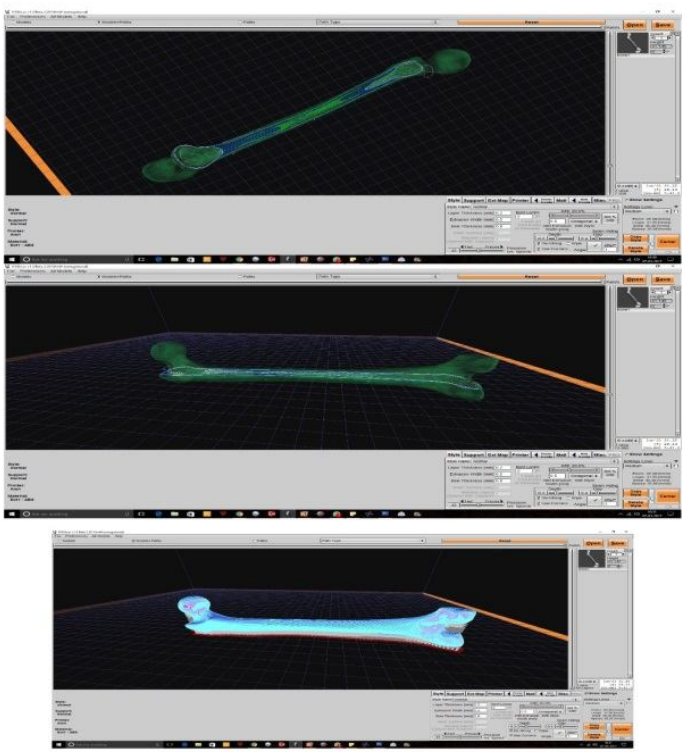
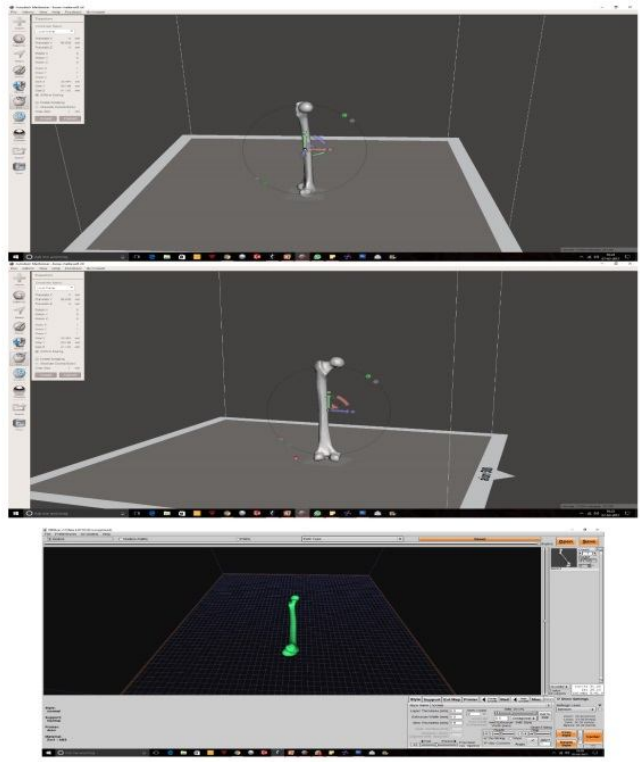

Fig 4: The figure shows Design of Bio Medical Bone

We made femur bone of 8 inches long horizontally at a temperature of 2300c. science has been developed now -a-days human organs like liver, ears are also $3 \mathrm{~d}$ printed using biomaterials and human tissues. Recently, in Russia a person met with a fatal accident and his skull has been damaged and by using additive manufacturing it has been repaire

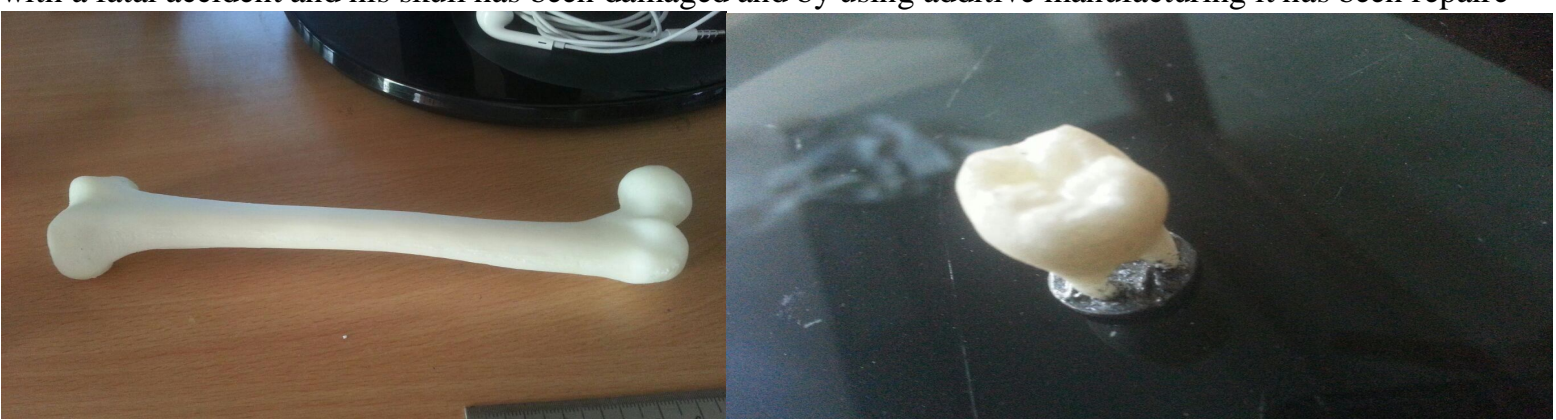

Fig 5: The figure shows Fabricated Bio Medical Bone and Teeth Using Additive Manufacturing

\section{ENGINEERING ANALYSIS}

\subsection{Human Bone strength Evaluation through different Mechanical Tests}

In human anatomy, the femur is the longest and largest bone. Along with the temporal bone of the skull, it is one of the two strongest bones in the body. The average adult male femur is 48 centimeters (18.9 in) in length and $2.34 \mathrm{~cm}(0.92 \mathrm{in})$ in diameter and can support up to 2 times the weight of an adult. It forms part of the hip joint (at the acetabulum) and part of the knee joint, which is located above. There are four eminences, or protuberances, in the human femur: the head, the 
greater trochanter, the lesser trochanter, and the lower extremity. They appear at various times from just before birth to about age 14 .

Bone density (or bone mineral density) is a medical term normally referring to the amount of mineral matter per square centimeter of bones. Bone density (or BMD) is used in clinical medicine as an indirect indicator of osteoporosis and fracture risk.This medical bone density is not the true physical density of the bone, which would be computed as mass per volume. It is measured by a procedure called densitometry, often performed in the radiology or nuclear medicine departments of hospitals or clinics. The measurement is painless and non-invasive and involves low radiation exposure. Measurements are most commonly made over the lumbar spine and the upper part of the hip. Average density is around $1500 \mathrm{~kg} \mathrm{~m} 3$.

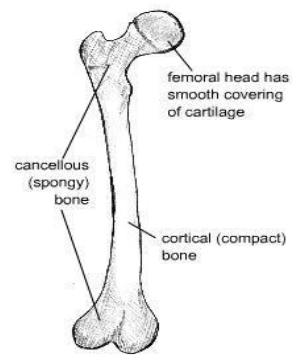

The DXA scan is typically used to diagnose and follow osteoporosis which is sensitive to certain metabolic diseases of bones in which bones are attempting to heal from infections, fractures, or tumors.

4.2 Mechanical properties of cortical bone

a. Bone Density

The material density of cortical bone is the wet weight divided by the specimen volume. It is a function of both the porosity and mineralization of the bone materials. Cortical bone has an average apparent density of approximately $1.9 \mathrm{~g} / \mathrm{cm} 3$.For cortical bone, apparent density and material density are basically the same, as there is no marrow space in compact bone. Therefore, cortical bone density is commonly used to describe the density of cortical bone. There is a positive correlation between apparent density of cortical bone and its mechanical properties. The true meaning of bone mineral density (BMD) is bone mineral mass per unit bone volume, or ash density if an ashing (or burning) method is used. Similarly, the true meaning of bone mineral content (BMC) describes the ratio of unit weight of the mineral portion to dry bone unit weight and is frequently reported as a percentage. BMD and BMC are positively correlated with the strength and stiffness of various bones, such as human ulna, human femur and tibia, bovine femur and tibia,32,46 feline femur,49 and a wide variety of animal bones.50 Many reports have shown linear or exponential increases in bone stiffness with increasing mineralization, such as the one proposed by Schaffler and Burr:

$\mathrm{E}=89.1 \mathrm{M} 391$

Where $\mathrm{E}$ is compressive elastic modulus and $\mathrm{M}$ is mineralization of bovine cortical bone

\section{b. Porosity}

The strong effects of porosity of cortical bone on mechanical properties have been well studied. It is easy to understand that a more porous bone has a weaker mechanical strength. Porosity (p) is defined as the ratio of void volume to total volume, which is commonly measured on two dimensional histologic sections (traditionally point counting)29,46 or X rays.55 In cortical bone, the mechanical properties are affected by Haversion canals and related resorption cavities and vascular channels. There are reports on the correlations of porosity and mechanical properties, such as the equation proposed by Schaffler and Burr on bovine cortical bone using tensile tests:

$\mathrm{E}=33.9(1-\mathrm{p}) 10.9$

Where $\mathrm{E}$ is the elastic modulus and $(1-\mathrm{p})$ is the bone volume fraction, and the equation by Currey for cortical bone of a wide variety of species under tension is

$\mathrm{E}=23.4(1-\mathrm{p}) 5.74$

McElhaney et al found that $\mathrm{E}=12.4(1-\mathrm{p}) 3$

\subsection{Mechanical properties of cancellous bone}

\subsubsection{Structural Properties}

The structural properties of cancellous bone are commonly measured by compression, tensile, or bending tests. The common phrase mechanical properties of cancellous bone means the structural properties. It is known that the strength and elastic modulus by tensile tests are smaller than that by compression tests. For example, the strength by tensile test is 
approximately $60 \%$ of the value by compression test reported by Kaplan et al.and the elastic modulus by tensile test is approximately $70 \%$ of the value by compression test reported by Keaveny et al.According, the values of strength and elastic moduli of cancellous bone are 1.5 to $38 \mathrm{MPa}$ and 10 to $1570 \mathrm{MPa}$, respectively. The structural properties of cancellous bone are much smaller than those of cortical bone. The average values of elastic modulus are several hundred mega Pascal for cancellous bone, compared with 5 to $21 \mathrm{GPa}$ for cortical bone.

\subsubsection{Bone Density}

There is a strong correlation between the mechanical properties of cancellous bone, both for strength and stiffness, and its apparent density and mineral (or ash) density. The apparent density of cancellous bone ranges from 0.14 to $1.10 \mathrm{~g} / \mathrm{cm} 3$ (average: $0.62 \mathrm{~g} / \mathrm{cm} 3, \mathrm{n}=16$; . The compressive strength (6 in $\mathrm{MPa}$ ) of cancellous bone is related to its apparent density by a power law of the form:

The compressive modulus ( $\mathrm{E}$ in $\mathrm{MPa}$ ) of cancellous bone is related to the apparent density ed data of ash densities of human and animal cancellous bones are a they range from 0.19 to $0.56 \mathrm{~g} / \mathrm{cm} 3 \mathrm{with}$ an average of $0.37 \pm 0.10 \mathrm{~g} / \mathrm{cm} 3(\mathrm{n}=12)$, which is about $60 \%$ of the value of apparent density as shown in the following.

\subsection{Mechanical Testing Methods}

Mechanical testing studies of bone have been directed at determining the mechanical properties of whole bone and bone tissue under different loading conditions. In general, determination of mechanical properties of bone is done by the same methods used to study similar properties in metal, woods, and other structural materials and composites. These methods are based on fundamental principles of mechanics.

\subsubsection{Tensile Testing}

Tensile testing can be one of the most accurate methods for measuring bone properties, but bone specimens must be relatively large and should be carefully machined. Tensile test specimens for cortical bone and cancellous bone. Dimensions are derived from ASTM standard the ratio d/D should be around $1 / 2$ and the parallel length of the narrow section should be at least three times the size of the gauge diameter.

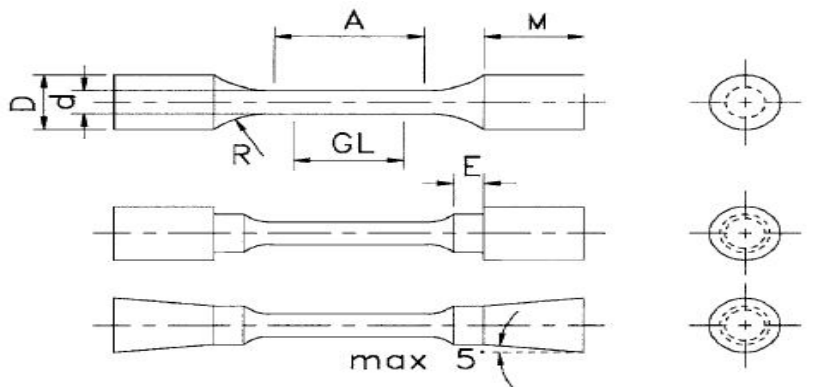

\subsubsection{Compressive Testing}

Fig 6. Tensile test specimen geometry for cortical bone tests.

Compression testing of bone specimens is a popular technique, especially for cortical bone because relatively small specimens can be used. Compressive tests, however, tend to be less accurate than tensile tests due to friction and compression-platen end effects imposed on the bone specimen during the test.

\subsubsection{Four-Point Bending}

Four-point bending occurs when two force couples acting on a structure produce two equal moments. A force couple refers to a pair of parallel forces of equal magnitude but opposite direction applied to a structure .The bending moment magnitude is the same throughout the area between the force couples; hence, the structure being tested should fracture at its weakest point.

\subsubsection{Torsional Testing}

A diaphyseal segment from a long bone might be grossly approximated as a hollow cylindrical shaft made from a homogeneous, linear elastic material. Such a shaft might have a certain inner radius, ri, an outer radius, ro, and a length, L. If one end, A, of the shaft is fixed, and a torsional force, T, is applied to the opposite end, B, then end B will rotate in its own plane through some angle with respect to end $\mathrm{A}$, the theoretical torsional share stress can be calculate by using equation.

\section{ANALYSIS OF HUMAN FEMUR BONE USING ANSYS WORKBENCH 5.1 ENGINEERING DATA} Material used: HYDROXYAPATITE

\begin{tabular}{|l|c|}
\hline \multicolumn{1}{|c|}{ PARAMETERS } & VALUES \\
\hline DENSITY & $1900 \mathrm{Kg} / \mathrm{m} 3$ \\
\hline YOUNG'S MODULUS & $14700 \mathrm{Mpa}$ \\
\hline
\end{tabular}




\begin{tabular}{|l|c|}
\hline POISSON'S RATIO & 0.3 \\
\hline TENSILE LOAD & $550 \mathrm{Mpa}$ \\
\hline COMPRESSION RATIO & $1500 \mathrm{Mpa}$ \\
\hline TEMPERATURE & Nearly body temperature \\
\hline
\end{tabular}

Using ANSYS workbench software following parameters are found out.

1. Equivalent stress

2. Equivalent Elastic Strain

3. Bone deformation

Firstly a file of femur bone is loaded and applying standard engineering data and by fixing at one corner of femur bone and at another end by adding compression stress the following parameters are determined.

One end is to be fixed because we can't loads on two sides of bone as compression is obtained on socket of femur bone and other side is fixed.

5.2 RESULTS:

Basing on the analysis made on ANSYS workbench following parameters are found out.

\begin{tabular}{|l|l|}
\hline ENGINEERING PARAMETERS & VALUES \\
\hline MAX. COMPRESSIVE STRESS & $0.811 \mathrm{MPA}$ \\
\hline ELASTIC STRAIN & 0.0015437 \\
\hline TOTAL DEFORMATION & $6.3342 \mathrm{~mm}$ \\
\hline
\end{tabular}

\section{VII.CONCLUSIONS:}

The outstanding feature of all AM techniques is their capability to produce parts of high geometrical complexity which cannot be manufactured by any other production technique. This works because of the tool-free layer-by-layer approach of all AM processes. Parts are produced based on 3D-CAD-model-data without any tooling needed. The number of available materials is still limited compared to other processes such as milling or injection moulding, but the number of materials qualified for polymer and metal based processes is growing. Many AM techniques offer part qualities which are comparable to those resulting from conventional manufacturing methods.

The AM produced parts can be used and post processed (milled, drilled, coated) like any other standard industrial part. Especially in metal, AM produced parts often exceed some of the mechanical property values of those machined from standard bulk material. Another benefit is the outstanding material efficiency of most AM processes. Scrap rates for AM parts are usually below 5\%, compared to scrap rates of more than $90 \%$ with many complex milled parts. With a decline in available raw material and rising costs this material efficiency will remain a major advantage in the long term.

Looking to the future, it can be confidently predicted that AM is set to achieve an increasing market share of production processes, helped with the introduction of faster systems with more powerful lasers and larger building chambers. A significant number of materials will be qualified for AM and over time multi-material systems for many of the processes will become available.

\section{REFERENCES}

[1]. Gaytan SM, Murr LE, Martinez E, Martinez JL, Machado BI, Ramirez DA, Medina F, Collins

[2]. Wicker RB (2010) Comparison of microstructures and mechanical properties for solid and mesh cobalt-base alloy prototypes fabricated by electron beam melting. Metall Mater Trans 41A:3216-3227

[3]. Murr LE, Martinez E, Gaytan SM, Ramirez DA, Machado BI, Shindo PW, Martinez JL, Medina F,

[4]. Wooten J, Ciscel D, Ackelid U, Wicker RB (2011) Microstructural architecture, microstructures and mechanical properties for a nickel-base superalloy fabricated by electron beammelting. Metall Mater Trans A 42A:3491-3508

[5]. Aguilar Z (2012) Nanomaterials for medical applications. Elsevier, New York

[6]. Alleman JE, Mossman BT (1997) Asbestos revisited. Sci Am 7:70-78

[7]. DeVolder MFL, Towfick JH, Boughman RH, Hart AJ (2013) Carbon nanotubes: present and future commercial applications. Science 339:535-539 (with supplementary online material)

[8]. Garza KM, Soto KF, Murr LE (2008) Cytotoxicity and reactive oxygen species generation from aggregated carbon and carbonaceous nanoparticulate materials. Int J Nanomedicine 3(1):1-12 